\title{
Novel real-time joint-transform correlation by use of acousto-optic heterodyning
}

\author{
Ting-Chung Poon and Ying Qi
}

\begin{abstract}
To replace the film recording aspect of performing optical correlation, conventional real-time jointtransform correlation (JTC) optical systems make use of a spatial light modulator (SLM) located in the Fourier plane to record the joint-transform power spectrum (JPS) to achieve real-time processing. The use of an SLM in the Fourier plane, however, is a major drawback in these systems because SLMs are limited in resolution, phase uniformity, and contrast ratio, which are, therefore, not desirable for robust applications. We propose a hybrid (optical/electronic) processing technique to achieve real-time jointtransform correlation. The technique employs acousto-optic heterodyning scanning. The proposed real-time JTC system does not require an SLM at the Fourier plane as in other real-time JTC systems. This departure from the conventional scheme is extremely important as the proposed approach does not depend on SLM issues. We develop the theory of the technique and substantiate it with experimental results. (C) 2003 Optical Society of America
\end{abstract}

OCIS codes: $\quad 070.0070,070.4550,070.5010$.

\section{Introduction and Background}

The correlation of two images is one of the most important mathematical operations in image processing and pattern recognition. ${ }^{1,2}$ As the images become more and more complex and large in size, the calculation becomes time consuming for a digital computer. Optical processing is an alternative to digital processing because it offers greater speed. Parallel optical processing by use of frequency domain architecture is fastest, but often lacks flexibility and accuracy. Scanning optical processing is fast, accurate, and flexible. ${ }^{3}$ In this paper, we combine the scanning optical technique with electronic processing to achieve real-time joint-transform correlation (JTC) for image recognition purposes. The system is hybrid (optical/electronic) in nature and its advantage is that the speed and data acquisition rate of such a hybrid optical-electronic processing can be made compatible with that of subsequent digital or optical signal processing. In what follows in this section we give a brief review of conventional real-time JTC systems and point out some of

The authors are with the Optical Image Processing Laboratory, Bradley Department of Electrical and Computer Engineering, Virginia Polytechnic Institute and State University, Blacksburg, Virginia 24061.

Received 1 November 2002; revised manuscript received 16 April 2003.

0003-6935/03/234663-07\$15.00/0

(C) 2003 Optical Society of America their major drawbacks. In Section 2 we describe a general theory of the hybrid optical system, which employs the theory of optical heterodyne scanning. In Section 3 we show that when a chirp grating is optically scanned, we can perform the cross correlation of two patterns without the major drawbacks encountered in conventional real-time JTC systems. In Section 4 we develop the optical system in the context of JTC. A practical optical implementation of the hybrid system is shown and described in Section 5 and experimental results are demonstrated. In Section 6 we make concluding remarks.

Owing to the advent of spatial light modulators (SLMs), ${ }^{4}$ real-time or video-rate joint-transform correlation systems have been accomplished by directly writing the reference object and the target object on SLMs, and the detection of the so-called jointtransform power spectrum (JPS) is subsequently performed by another SLM, which may be coherently read out for correlation operation. ${ }^{5-10}$ The conventional real-time JTC system is shown in Fig. 1. $g_{1}(x$, $y$ ) and $g_{2}(x, y)$ are the reference object and the target object, respectively. They are separated by the distance $2 x_{0}$ along the $x$-direction. The JPS, $J\left(k_{x}, k_{y}\right)$, on the back focal plane of lens $\mathrm{L}_{1}$ is then given by

$$
\begin{aligned}
J\left(k_{x}, k_{y}\right)= & \left|F\left\{g_{1}\left(x-x_{0}, y\right)\right\}+F\left\{g_{2}\left(x+x_{0}, y\right)\right\}\right|^{2} \\
= & \left|G_{1}\left(k_{x}, k_{y}\right)\right|^{2}+\left|G_{2}\left(k_{x}, k_{y}\right)\right|^{2} \\
& +G_{1}^{*}\left(k_{x}, k_{y}\right) G_{2}\left(k_{x}, k_{y}\right) \exp \left(j 2 k_{x} x_{0}\right) \\
& +G_{1}\left(k_{x}, k_{y}\right) G_{2}^{*}\left(k_{x}, k_{y}\right) \exp \left(-j 2 k_{x} x_{0}\right),
\end{aligned}
$$




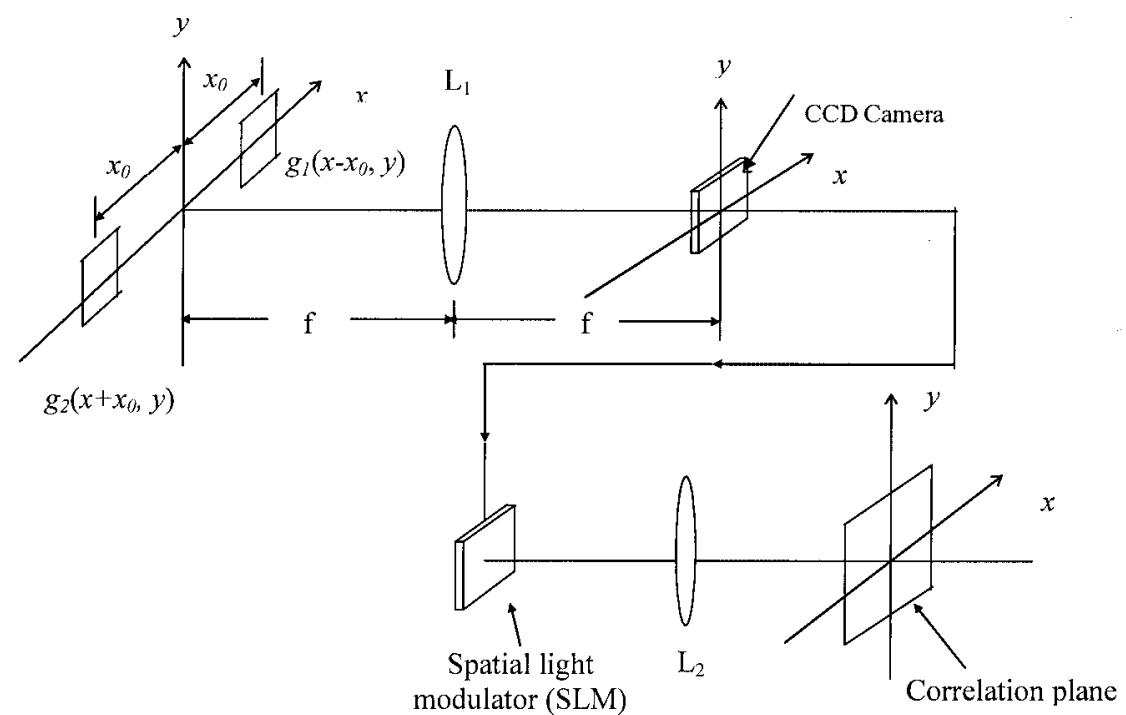

Fig. 1. Conventional real-time JTC system.

where $G_{\mathrm{i}}\left(k_{x}, k_{y}\right)$ is the Fourier transform of the function $g_{i}(x, y)$ for $i=1,2$, and is given by

$$
\begin{aligned}
G_{i}\left(k_{x}, k_{y}\right) & =\iint g_{i}(x, y) \exp \left[j\left(k_{x} x+k_{y} y\right)\right] \mathrm{d} x \mathrm{~d} y \\
& =F\left\{g_{i}(x, y)\right\}_{k_{x}, k_{y}},
\end{aligned}
$$

with $k_{x}=k_{0} x / f$ and $k_{y}=k_{0} y / f$, where $f$ is the focal length of the lens $\mathrm{L}_{1}$ and $k_{0}$ is the wavenumber of the laser. In real-time JTC systems, the JTPS is detected by a SLM, e.g., a CCD camera and the output of the CCD camera is fed to another SLM for coherent display as illustrated in Fig. 1, where lens $\mathrm{L}_{2}$ is a Fourier transform lens with the same focal length as lens $\mathrm{L}_{1}$. Upon coherent display on the correlation plane we have the Fourier transform of $J\left(k_{x}, k_{y}\right)$ :

$$
\begin{aligned}
F\left\{J\left(\frac{k_{0} x}{f}, \frac{k_{0} y}{f}\right)\right\}_{k_{x}, k_{y}}= & C_{11}(-x,-y)+C_{22}(-x,-y) \\
& +C_{12}\left(-x-2 x_{0},-y\right) \\
& +C_{21}\left(-x+2 x_{0},-y\right),
\end{aligned}
$$

where

$$
\begin{aligned}
C_{i j}(x, y)= & g_{i}(x, y) \odot g_{j}(x, y)=\iint g_{i}^{*}\left(x^{\prime}, y^{\prime}\right) \\
& \times g_{j}\left(x+x^{\prime}, y+y^{\prime}\right) \mathrm{d} x^{\prime} \mathrm{d} y^{\prime},
\end{aligned}
$$

with $i=1$ or 2 , and $j=1$ or 2 . The symbol $\odot$ denotes correlation involving coordinates $x$ and $y . \quad C_{i j}(x, y)$ is the autocorrelation when $i=j$, and is the cross correlation when $i \neq j$. Hence if the target and the reference objects are the same, besides a strong peak at the origin of the correlation plane due to the first two terms of Eq. (3), we have two strong peaks centered at $x= \pm 2 x_{0}$ due to the last two terms of Eq. (3).

There are two major drawbacks with conventional real-time systems. Owing to the existence of zeroth- order spectra, i.e., the first two terms in Eq. (1), a physical separation between the reference object and the target object is required.11 This requirement hampers the utilization efficiency of the input spatial domain and lowers the diffraction efficiency of the correlation peaks, and attempts have been made to alleviate this problem. ${ }^{12-26}$ Another major drawback is the need for a high quality SLM, such as with a high spatial resolution requirement, for coherent display of JPS to obtain correlation output. This is clear by inspecting the last two terms of Eq. (1), as

$$
\begin{aligned}
& G_{1}^{*}\left(k_{x}, k_{y}\right) G_{2}\left(k_{x}, k_{y}\right) \exp \left(j 2 k_{x} x_{0}\right) \\
& \quad+G_{1}\left(k_{x}, k_{y}\right) G_{2}^{*}\left(k_{x}, k_{y}\right) \exp \left(-j 2 k_{x} x_{0}\right) \\
& =2\left|G_{1}\left(k_{x}, k_{y}\right) G_{2}\left(k_{x}, k_{y}\right)\right| \cos \left(2 k_{x} x_{0}+\theta\right),
\end{aligned}
$$

where $\theta$ is the phase angle of $G_{1}^{*} G_{2}$. We see that the correlation information is carried by a spatial carrier with frequency $2 x_{0} / \lambda f$, where $\lambda=2 \pi / k_{0}$ is the wavelength of light, and we have made use of $k_{x}=k_{0} x / f$ into the argument of the cosine term to find the frequency of the spatial carrier. For $2 x_{0}=30 \mathrm{~mm}, \lambda=$ $0.6 \mu \mathrm{m}$, and $f=50 \mathrm{~cm}$, the required spatial resolution of the SLM is $2 x_{0} / \lambda f \sim 100$ cycle $/ \mathrm{mm}$. Very often in practice, $2 x_{0}$ is made to be small enough or the focal length $f$ is made large enough so that the CCD camera or the subsequent SLM can resolve the spatial carrier. Hence the spatial resolution requirement of the CCD and SLM should be in general high, i.e., the CCD camera and the subsequent SLM must be able to resolve these fine details to have the real-time system work. In addition, phase uniformity and contrast ratio of the SLM also are important considerations. In the next section we propose a hybrid optical/electronic system that can perform real-time correlation and yet does not involve these critical drawbacks. Specifically, there is no need to use an SLM for coherent display of JPS in the present proposed system. 


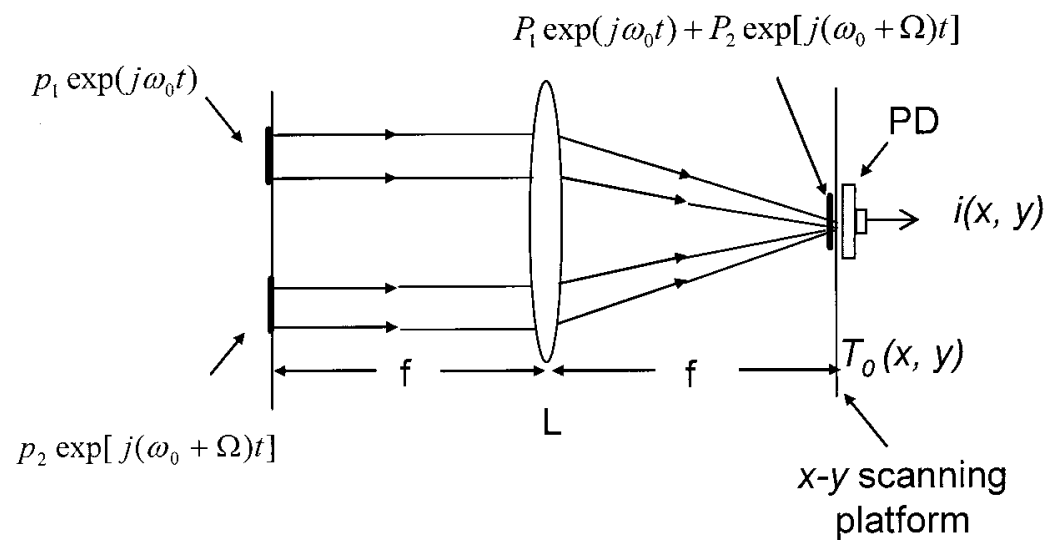

Fig. 2. Basic optical heterodyne scanning system.

\section{Theory of Optical Heterodyne Scanning}

In this section we briefly review the theory of optical heterodyne scanning originally developed by Poon and Korpel. ${ }^{27}$ With some modification, the system discussed is used in the next section to perform a real-time correlation operation. A typical heterodyne optical scanning system is shown in Fig. 2. A collimated laser at temporal frequency $\omega_{0}$ is used to illuminate a transparency function, $p_{1}(x, y)$, located on the front focal plane of lens $\mathrm{L}$ with focal length $f$. The transparency function is usually called the pupil function. ${ }^{27-30}$ The other pupil, $p_{2}(x, y)$, also located on the same focal plane, is illuminated by a laser of temporal frequency $\omega_{0}+\Omega$. The laser's temporal frequency offset of $\Omega$ can be introduced, for example, by an acousto-optic modulator. ${ }^{31}$ The combined optical scanning field on the focal plane is given by

$$
\begin{aligned}
P_{1}\left(\frac{k_{0} x}{f}, \frac{k_{0} y}{f}\right) & \exp \left(j \omega_{0} t\right)+P_{2}\left(\frac{k_{0} x}{f}, \frac{k_{0} y}{f}\right) \\
& \times \exp \left[j\left(\omega_{0}+\Omega\right) t\right],
\end{aligned}
$$

where $P_{i}\left(k_{0} x / f, k_{0} y / f\right)=F\left\{p_{i}(x, y)\right\} \frac{k_{0} x}{f}, \frac{k_{0} y}{f}$ and $i=1,2$. The combined optical field or the scanning pattern is used to two-dimensional raster scan a target with amplitude distribution given by $T_{o}(x, y)$ located on the back focal plane of lens L. Alternatively, the target can be placed on a $x-y$ scanner platform, as shown in Fig. 2, while the optical beam is stationary. The photodetector, which responds to the intensity of the optical transmitted field or scattered field, generates a current given by

$$
\begin{aligned}
i(x, y)= & \iint_{A} \mid\left\{P_{1}\left(\frac{k_{0} x^{\prime}}{f}, \frac{k_{0} y^{\prime}}{f}\right) \exp \left(j \omega_{0} t\right)\right. \\
& \left.+P_{2}\left(\frac{k_{0} x^{\prime}}{f}, \frac{k_{0} y^{\prime}}{f}\right) \exp \left[j\left(\omega_{0}+\Omega\right) t\right]\right\} \\
& \times\left. T_{o}\left(x+x^{\prime}, y+y^{\prime}\right)\right|^{2} \mathrm{~d} x^{\prime} \mathrm{d} y^{\prime} .
\end{aligned}
$$

Note that the integration is over the area $A$ of the photodetector, and $x=x(\mathrm{t})$ and $y=y(\mathrm{t})$ represent the instantaneous position of the scanning pattern, and the shifted coordinates of $T_{o}$ represent the action of scanning. Note also that the current contains the baseband current and the heterodyne current at the temporal frequency $\Omega$. The heterodyne current, which contains the useful information, is given by

$$
\begin{aligned}
i_{\Omega}(x, y)= & \operatorname{Re}\left[\iint_{A} P_{1}^{*}\left(\frac{k_{0} x^{\prime}}{f}, \frac{k_{0} y^{\prime}}{f}\right) P_{2}\left(\frac{k_{0} x^{\prime}}{f}, \frac{k_{0} y^{\prime}}{f}\right)\right. \\
& \left.\times\left|T_{o}\left(x+x^{\prime}, y+y^{\prime}\right)\right|^{2} \mathrm{~d} x^{\prime} \mathrm{d} y^{\prime} \exp (j \Omega t)\right],
\end{aligned}
$$

where we have adopted the convention for phasor $\psi_{p}$ as $\psi(x, y, t)=\operatorname{Re}\left[\psi_{p}(x, y, t) \exp (j \Omega t)\right]$, and where $\operatorname{Re}[$. denotes the real part of the content inside the brackets. Equation (8) can be written as

$$
i_{\Omega}(x, y)=\operatorname{Re}\left[i_{\Omega_{p}}(x, y) \exp (j \Omega t)\right]
$$

where

$$
\begin{aligned}
i_{\Omega_{p}}(x, y)= & \iint_{A} P_{1}^{*}\left(\frac{k_{0} x^{\prime}}{f}, \frac{k_{0} y^{\prime}}{f}\right) P_{2}\left(\frac{k_{0} x^{\prime}}{f}, \frac{x_{0} y^{\prime}}{f}\right) \\
& \times\left|T_{o}\left(x+x^{\prime} y+y^{\prime}\right)\right|^{2} \mathrm{~d} x^{\prime} \mathrm{d} y^{\prime}
\end{aligned}
$$

is the output phasor that denotes the amplitude and the phase information of the heterodyne current that constitute the scanned and processed version of the target $\left|T_{\mathrm{o}}\right|^{2}{ }^{27,29} \quad$ Note that the optical scanning system under consideration can process the intensity distribution of the object being scanned. The system has been used for the so-called two-pupil synthesis for bipolar or even complex incoherent image processing. ${ }^{27-30}$

\section{Realization of Real-Time Correlation}

In this Section we show how a one-dimensional (1-D) scanning of a chirp grating will result in the correlation of the two pupils presented in the system. We therefore let $\left|T_{\mathrm{o}}\right|^{2}=1+\cos \left(a x^{2}\right)$, where $a$ is a grating 
constant. Since $2 \cos \left(a x^{2}\right)=\exp \left(j a x^{2}\right)+\exp \left(-j a x^{2}\right)$, Eq. (9b) becomes

$$
\begin{aligned}
i_{\Omega_{p}}(x)= & \iint_{A} P_{1}^{*}\left(\frac{k_{0} x^{\prime}}{f}, \frac{k_{0} y^{\prime}}{f}\right) P_{2}\left(\frac{k_{0} x^{\prime}}{f}, \frac{x_{0} y^{\prime}}{f}\right) \\
& \times\left\{1+\frac{1}{2} \exp \left[j a\left(x+x^{\prime}\right)^{2}\right]\right. \\
& \left.+\frac{1}{2} \exp \left[-j a\left(x+x^{\prime}\right)^{2}\right]\right\} \mathrm{d} x^{\prime} \mathrm{d} y^{\prime} \\
= & I_{0}+\frac{1}{2} t_{1}(x)+\frac{1}{2} t_{2}(x),
\end{aligned}
$$

where

$$
\begin{aligned}
I_{0}= & \iint_{A} P_{1}^{*}\left(\frac{k_{0} x^{\prime}}{f}, \frac{k_{0} y^{\prime}}{f}\right) P_{2}\left(\frac{k_{0} x^{\prime}}{f}, \frac{k_{0} y^{\prime}}{f}\right) \\
& \times \mathrm{d} x^{\prime} \mathrm{d} y^{\prime}, \\
t_{1}(x)= & \iint_{A} P_{1}^{*}\left(\frac{k_{0} x^{\prime}}{f}, \frac{k_{0} y^{\prime}}{f}\right) P_{2}\left(\frac{k_{0} x^{\prime}}{f}, \frac{k_{0} y^{\prime}}{f}\right) \\
& \times \exp \left[j a\left(x+x^{\prime}\right)^{2} \mathrm{~d} x^{\prime} \mathrm{d} y^{\prime},\right.
\end{aligned}
$$

and

$$
\begin{aligned}
t_{2}(x)= & \iint_{A} P_{1}^{*}\left(\frac{k_{0} x^{\prime}}{f}, \frac{k_{0} y^{\prime}}{f}\right) P_{2}\left(\frac{k_{0} x^{\prime}}{f}, \frac{k_{0} y^{\prime}}{f}\right) \\
& \times \exp \left[-j a\left(x+x^{\prime}\right)^{2}\right] \mathrm{d} x^{\prime} \mathrm{d} y^{\prime},
\end{aligned}
$$

We see that the first term $I_{0}$ is some complex constant, and we shall manipulate the second term given by Eq. (10b). By expanding the exponential term and rearranging, Eq. (10b) becomes

$$
\begin{aligned}
t_{1}(x)= & \exp \left(j a x^{2}\right) \iint_{A} P_{1}^{*}\left(\frac{k_{0} x^{\prime}}{f}, \frac{k_{0} y^{\prime}}{f}\right) P_{2}\left(\frac{k_{0} x^{\prime}}{f}, \frac{k_{0} y^{\prime}}{f}\right) \\
& \times \exp \left(j a x^{\prime 2}\right) \exp \left(j 2 a x x^{\prime}\right) \mathrm{d} x^{\prime} \mathrm{d} y^{\prime} \\
= & \exp \left(j a x^{2}\right) F\left\{P_{1}^{*}\left(\frac{k_{0} x}{f}, \frac{k_{0} y}{f}\right) P_{2}\left(\frac{k_{0} x}{f}, \frac{k_{0} y}{f}\right)\right. \\
& \left.\times \exp \left(j a x^{2}\right)\right\}_{k_{x}=2 a x, k_{y}=0}
\end{aligned}
$$

At this point, we are making an assumption in that the grating period, a, is small such that Eq. (11) becomes

$$
\begin{aligned}
t_{1}(x)= & \exp \left(j a x^{2}\right) F\left\{P_{1}^{*}\left(\frac{k_{0} x}{f}, \frac{k_{0} y}{f}\right)\right. \\
& \left.\times P_{2}\left(\frac{k_{0} x}{f}, \frac{k_{0} y}{f}\right)\right\}_{k_{x}=2 a x, k_{y}=0} .
\end{aligned}
$$

This assumption is reminiscent of the far-field approximation in diffraction in that the Fresnel diffraction becomes the Fraunhofer diffraction. ${ }^{32}$ We can now express Eq. (12), besides some constant, in terms of the correlation integral:

$$
\begin{aligned}
& t_{1}(x)=\exp \left(j a x^{2}\right) \\
& \quad\left[P_{1}\left(-\frac{f k_{x}}{k_{0}},-\frac{f k_{y}}{k_{0}}\right) \odot P_{2}\left(-\frac{f k_{x}}{k_{0}},-\frac{f k_{y}}{k_{0}}\right)\right]_{k_{x}=2 a x, k_{y}=0} .
\end{aligned}
$$

Similarly, Eq. (10c) becomes

$$
\begin{aligned}
t_{2}(x)= & \exp \left(-j a x^{2}\right)\left[P_{1}\left(\frac{f k_{x}}{k_{0}}, \frac{f k_{y}}{k_{0}}\right)\right. \\
& \left.\odot P_{2}\left(\frac{f k_{x}}{k_{0}}, \frac{f k_{y}}{k_{0}}\right)\right]_{k_{x}=2 a x, k_{y}=0} .
\end{aligned}
$$

Putting these results into Eq. (9a), we have

$$
i_{\Omega}(x, y)=\operatorname{Re}\left[i_{\Omega_{p}}(x, y) \exp (j \Omega t)\right],
$$

where

$$
\begin{aligned}
i_{\Omega_{p}}(x)= & I_{0}+\frac{1}{2} \exp \left(j a x^{2}\right)\left[P_{1}\left(-\frac{f k_{x}}{k_{0}},-\frac{f k_{y}}{k_{0}}\right)\right. \\
& \left.\odot P_{2}\left(-\frac{f k_{x}}{k_{0}},-\frac{f k_{y}}{k_{0}}\right)\right]_{k_{x}=2 a x, k_{y}=0} \\
& +\frac{1}{2} \exp \left(-j a x^{2}\right)\left[p_{1}\left(\frac{f k_{x}}{k_{0}}, \frac{f k_{y}}{k_{0}}\right)\right. \\
& \left.\odot p_{2}\left(\frac{f k_{x}}{k_{0}}, \frac{f k_{y}}{k_{0}}\right)\right]_{k_{x}=2 a x, k_{y}=0}
\end{aligned}
$$

It is clear that if $p_{1}$ and $p_{2}$ represent patterns to be matched, we achieve the correlation of the two patterns.

\section{Real-Time Joint-Transform Correlation without use of SLM in the Focal Plane}

In this section we develop Eq. (15) in the context of JTC and describe an extraction scheme to display the correlation information. We let $p_{1}(x, y)=g_{1}\left(x-x_{0}\right.$, $y)$ and $p_{2}(x, y)=g_{2}\left(x+x_{0}, y\right)$, where $g_{1}(x, y)$ and $g_{2}(x$, $y$ ) are the two patterns to be matched, and they are now located side by side in the front focal plane of lens L as realistically shown in Fig. 2. Again, these patterns form a composite beam to 1-D scan the chirp grating. The heterodyne current given by Eq. (15) and with $p_{1}(x, y)$ and $p_{2}(x, y)$ given above, and using the definition of correlation in Eq. (4), we have, after some manipulations,

$$
\begin{aligned}
i_{\Omega_{p}}(x)= & I_{0}+\frac{1}{2} \exp \left(j a x^{2}\right) C_{12}\left(\frac{-2 a x f}{k_{0}}-2 x_{0}, 0\right) \\
& +\frac{1}{2} \exp \left(-j a x^{2}\right) C_{12}\left(\frac{2 a x f}{k_{0}}-2 x_{0}, 0\right),
\end{aligned}
$$




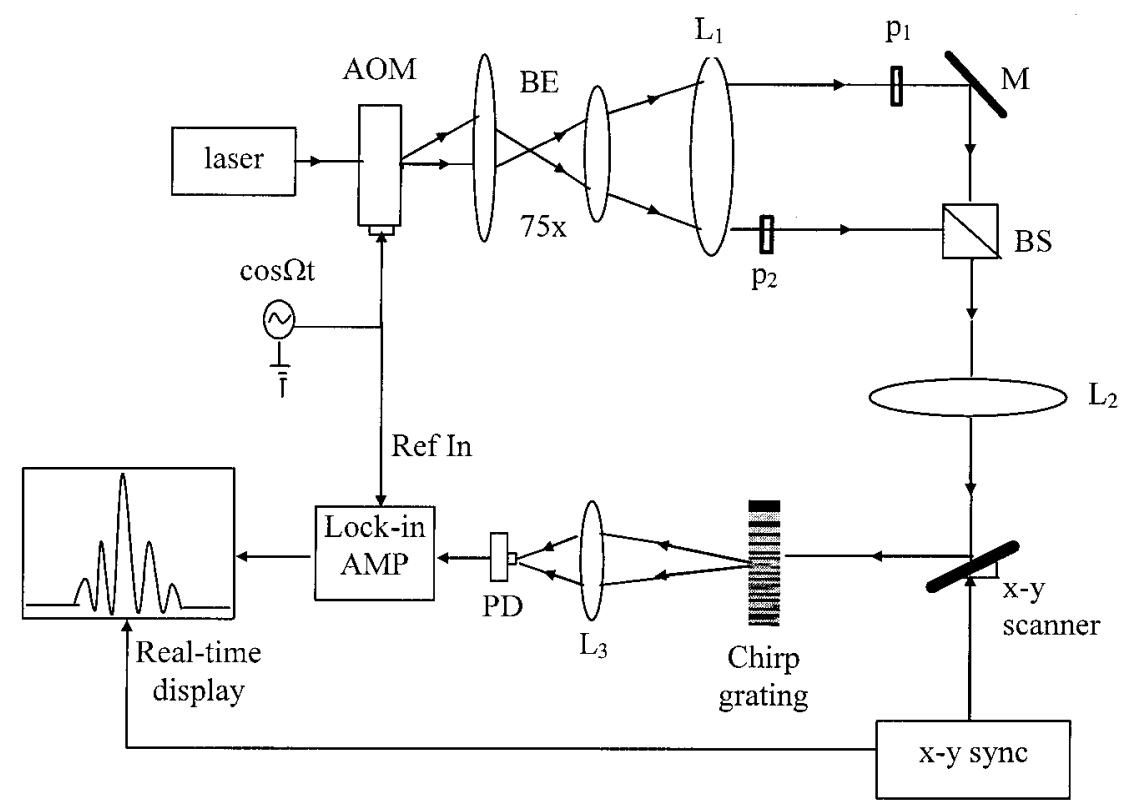

Fig. 3. Practical implementation of Fig. 2.

and Eq. (15a) becomes

$$
\begin{aligned}
i_{\Omega}(x)= & \left|I_{0}\right| \cos \left(\Omega t+\theta_{0}\right)+\frac{1}{2}\left|C_{12}\left(\frac{-2 a x f}{k_{0}}-2 x_{0}, 0\right)\right| \\
& \times \cos \left(\Omega t+a x^{2}+\theta_{1}\right) \\
& +\frac{1}{2}\left|C_{12}\left(\frac{2 a x f}{k_{0}}-2 x_{0}, 0\right)\right| \\
& \times \cos \left(\Omega t-a x^{2}+\theta_{2}\right),
\end{aligned}
$$

where $\theta_{0}, \theta_{1}$, and $\theta_{2}$ are the phase angles of $I_{0}$, $C_{12}\left(-2 a x f / k_{0}-2 x_{0}, 0\right)$, and $C_{12}\left(2 a x f / k_{0}-2 x_{0}, 0\right)$, respectively. Note that $i_{\Omega}(x)$ is a 1-D time signal as $x$ is a function of time, i.e., $x(t)$, and this signal is an amplitude modulated signal with carrier $\Omega$. The amplitude of the signal contains the correlation information, which can be extracted by use of a lock-in amplifier with a reference signal equal to $\cos (\Omega t)$. After the lock-in amplifier, from Eq. (17), we have

$$
\begin{array}{r}
i_{\text {lock-in }}(x) \propto\left|I_{0}\right| \cos \left(\theta_{0}\right)+\frac{1}{2}\left|C_{12}\left(\frac{-2 a x f}{k_{0}}-2 x_{0}, 0\right)\right| \\
\times \cos \left(a x^{2}+\theta_{1}\right)+\frac{1}{2}\left|C_{12}\left(\frac{2 a x f}{k_{0}}-2 x_{0}, 0\right)\right| \\
\times \cos \left(a x^{2}-\theta_{2}\right),
\end{array}
$$

which can be displayed with a real-time monitor such as an oscilloscope. If $x(t)=v t$, that is the scan position is linearly with time, where $v$ is the constant scanning speed of the laser beam, Eq. (18) then manifests itself as two cross correlations centered at two locations along the time scale: $t= \pm x_{0} k_{0} / v a f$, and of course, the correlations are amplitude modulated by the chirp-type functions $\cos \left[a(v t)^{2}+\theta_{1}\right]$ and $\cos \left[a(v t)^{2}\right.$ $\left.-\theta_{2}\right]$. If the two patterns are matched, there will be two correlation peaks and since the $y$ coordinates are zero within the correlation arguments, what we see is actually a line trace through the correlation peaks as $y=0$.

\section{Practical Implementation and Experimental Results}

Figure 3 shows the practical implementation of Fig. 2. The acousto-optic modulator operates in the Bragg regime ${ }^{31}$ at sound frequency $\Omega / 2 \pi \sim 40 \mathrm{MHz}$. The two diffracted beams, i.e., the zeroth-order beam and the first-order beam, are at frequencies $\omega_{0}$ and $\omega_{0}+\Omega$, respectively, where $\omega_{0}$ is the frequency of the laser light. After a $75 \times$ beam expander, the zerothorder beam is incident on the pattern $p_{1}$, and the first-order beam is incident on the other pattern $p_{2}$. The two patterns are located at the front focal plane of lens $\mathrm{L}_{2}$ of focal length approximately $50 \mathrm{~cm}$. They are then combined by the beam spltter projected through the $x-y$ scanner (in our case, only a 1-D scan along the $x$ direction is required as explained in the last section), and focused onto the chirp grating located at the back focal plane of lens $\mathrm{L}_{2}$. Lens $\mathrm{L}_{3}$ is used to collect all the light onto the photodetector. The output of the photodetector is then sent to the lock-in amplifier to give the current given by Eq. (18). If the two patterns are matched, strong peaks of the electrical current will be displayed on the real-time display such as an oscilloscope.

Experiments with different reference objects and target objects have been performed. To compare the results between different experiments, all the system parameters, such as the power of the laser source, the scan speed of $x-y$ scanner, the time constant and sensitivity of the lock-in amplifier, and the voltage scale and time scale of the oscilloscope are set to the same values throughout these experiments.

Figure 4 shows two identical animals (animal 1) of 


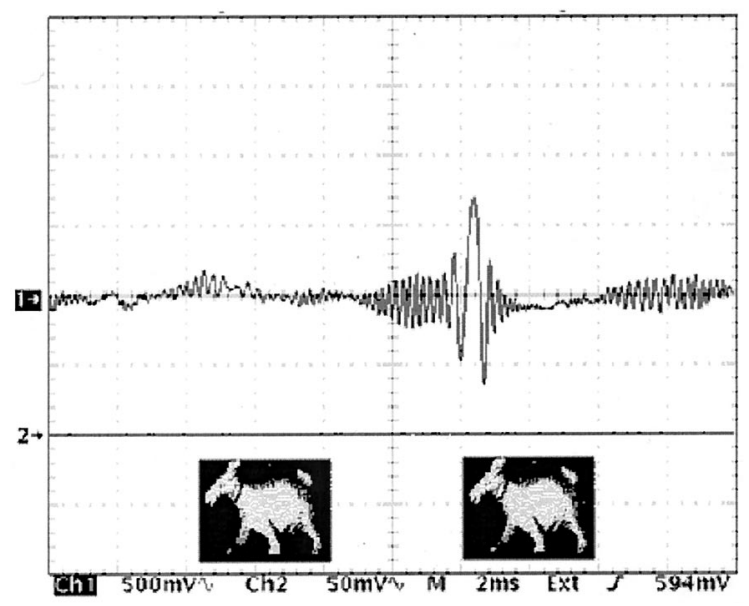

Fig. 4. Autocorrelation result of two identical animals (animal 1).

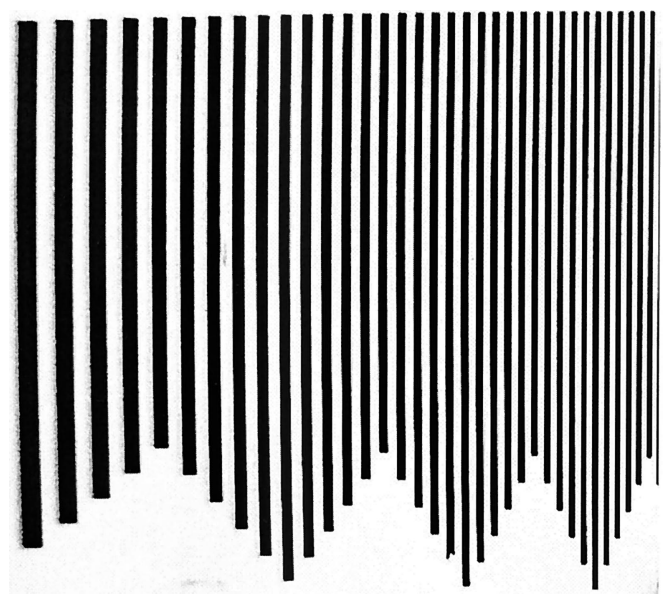

Fig. 5. Chirp grating used in the experiment.

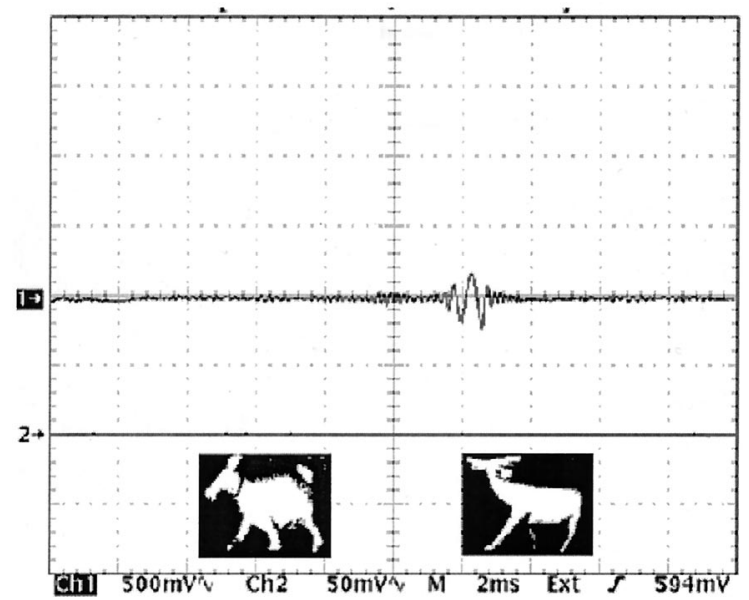

Fig. 6. Correlation result of two different animals.

the size of approximately 6 millimeters by 7 millimeters, shown on the bottom of the figure, which are placed at the front focal plane of lens $\mathrm{L}_{2}$ of a focal length of 50 centimeters for autocorrelation. The two patterns are separated by $2 x_{0} \approx 6$ millimeters,

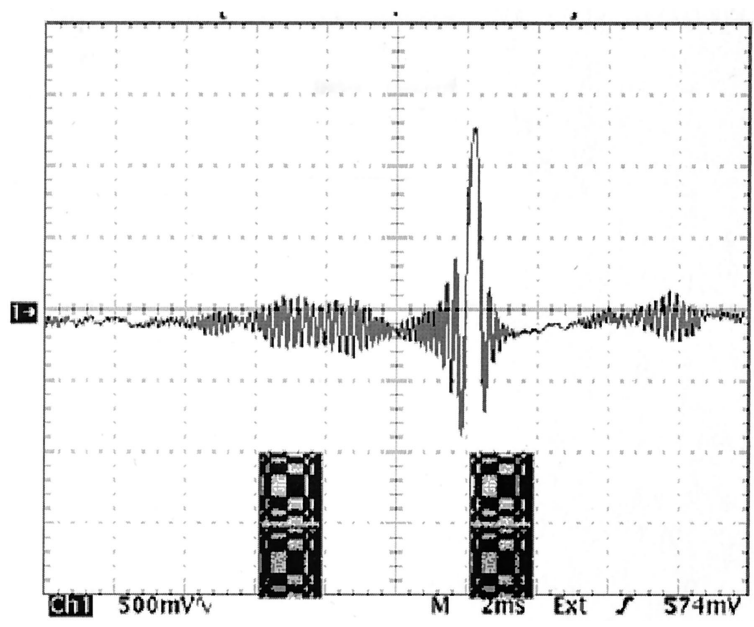

Fig. 7. Autocorrelation result of two identical random patterns.

which gives $2 x_{0} / \lambda f \sim 20$ cycle $/ \mathrm{mm}$. When a chirp grating is $1-\mathrm{D}$-scanned along the $x$ direction, a trace of electrical signal through the center of the autocorrelation will be displayed [see Eq. (18)]. Indeed, we see a strong peak display on the oscilloscope as shown in the figure. The time scale is 2 millisecond per division with a vertical scale of $500 \mathrm{mV}$ per division. Figure 5 shows the picture of a portion of the chirp grating used. It is basically a set of dark lines of varying separations printed on a glass substrate. The grating frequency ranges from 1 cycle/ $\mathrm{mm}$ to 25 cycle $/ \mathrm{mm}$. Because the grating used is not symmetrical, we only observe a single correlation peak.

In Figure 6, the cross correlation between target object (animal 2) and reference object (animal 1) is presented. The autocorrelation peak in Fig. 4 is approximately 3 times higher than the cross-correlation peak shown in Fig. 6. For patterns with more details, we can expect the autocorrelation peak to be stronger and sharper as is evident from Fig. 7, where two identical random patterns (3 millimeters by 8 millimeters are used. Figure 8 shows the cross cor-

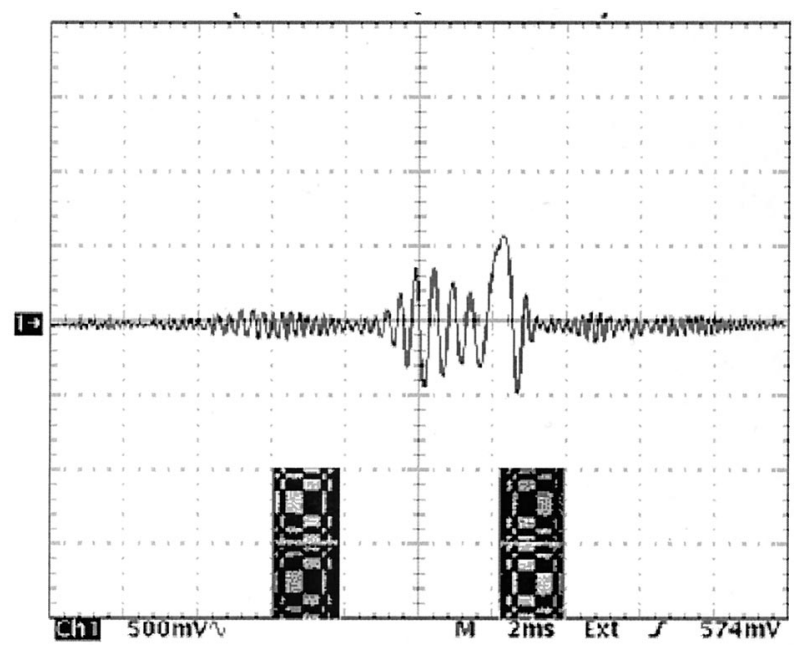

Fig. 8. Cross-correlation result of a random pattern and its contrast reversal pattern. 
relation when the random pattern and its contrast reversal pattern is used. The correlation peak is much lower than the one in Fig. 7 as expected.

In the proposed heterodyne scanning system the processing speed depends on how fast the optical scanner performs 1-D scanning. In our actual experiments, we have used a 1-D galvanometer with a scan rate of approximately $100 \mathrm{~Hz}$, corresponding to a scan time or processing speed of approximately 10 ms. For high processing speed of the order of microseconds and precise scanning, acousto-optic scanners could be used. ${ }^{31}$

\section{Concluding Remarks}

We have proposed what to our knowledge is a novel real-time joint-transform correlator without the use of any two-dimensional spatial light modulator located at the Fourier plane. The proposed system is hybrid (optical/electronic) and based on optical heterodyne scanning. It does not suffer from the two major drawbacks with the conventional real-time system: the existence of zeroth-order spectra (because of heterodyning) and the use of a high quality spatial light modulator for coherent display (because of the use of a chirp grating). This departure from the conventional scheme is extremely important as the proposed approach does not depend on SLM issues. Consequently, the proposed system should become more readily adapted to industry as well as military pattern-recognition applications.

As a final remark, we point out that the final output of our system is an electrical signal. It represents a line traced through the correlation peak [see Eq. (18)]. We therefore would expect the correlation peak to be as good as the one obtained from classical JTC systems. To obtain a higher correlation peak in our system, one can, for example, preprocess the input image such as the extraction of its edge information. ${ }^{3,33}$

We want to express our gratitude for the financial support by the National Science Foundation (ECS9810158). We also thank Guy Indebetouw for supplying us with some of the patterns used in the experiments.

\section{References}

1. A. VanderLugt, "Signal detection by complex spatial filter," IEEE Trans. Inf. Theory IT-10, 139-146 (1964).

2. C. S. Weaver and J. W. Goodman, "A technique for optically convolving two functions," Appl. Opt. 5, 1248-1249 (1966).

3. G. Indebetouw, "Scanning optical correlator," Opt. Lett. 6, 10-12 (1981).

4. T.-C. Poon, R. Juday, and T. Hara, eds., Feature Issue on Spatial Light Modulators, Appl. Opt. 37, 7471-7552 (1998).

5. J. E. Rau, "Real-time complex spatial modulation," J. Opt. Soc. Am. 57, 798-802 (1967).

6. P. Nisenson and R. A. Sprague, "Real-time optical correlation," Appl. Opt. 14, 2602-2606 (1975)

7. F. T. S. Yu and X. Lu, "A real-time programmable joint transform correlator," Opt. Commun 52, 10-16 (1984).

8. F. T. S. Yu, S. Jutamulia, T. W. Lin, and D. Gregory, "Adaptive real-time pattern recognition using a liquid crystal TV based joint transform correlator,” Appl. Opt. 26, 1370-1372 (1987).

9. J. M. Florence, "Joint-transform correlator systems using deformable-mirror spatial light modulator," Opt. Lett. 14, 341316 (1989).

10. T. D. Hudson, D. W. Trivett, D. Gregory, and J. C. Kirsch, "Real time optical correlator architectures using a deformable mirror spatial light modulator," Appl. Opt. 28, 4853-4860 (1989).

11. G. Lu, Z. Zhang, S. Wu, and F. T. S. Yu., "Implementation of a non-zero-order joint-transform correlator by use of phaseshifting techniques," Appl. Opt. 38, 470-483 (1995).

12. A. Gregory, "Time multiplexed miniature optical correlator," Lett. rep. RD-RE-88-02, U.S. Army Missile Command, Alabama (1988).

13. T. H. Bames, K. Matsuda, T. Eiju, K. Matsumoto, and F. Johnson, "Joint transform correlator using a phase only spatial light modulator," Jap. J. Appl. Phys. 29, L1293-L1296 (1990).

14. S. Jutamulia, G. M. Storti, D. A. Gregory, and J. C. Kirsch, "Illumination-independent high-efficiency joint transform correlator," Appl. Opt. 30, 4173-4175 (1991).

15. D. A. Gregory, J. C. Kirsch, and E. C. Tam, "Full complex modulation using liquid-crystal televisions," Appl. Opt. 31, 163-165 (1992).

16. Q. Tang and B. Javidi, "Technique for reducing the redundant and self-correlation terms in joint transform correlators," Appl. Opt. 32, 1911-1918 (1993).

17. M. S. Alam and M. A. Karim, "Fringe-adjusted joint transform correlation," Appl. Opt. 32, 4344-4350 (1993).

18. C. J. Kuo, "Joint transform correlator improved by means of the frequency-selective technique," Opt. Eng. 33, 522-527 (1994).

19. T. J. Grycewicz, "Applying time-modulation to the joint transform correlator," Opt. Eng. 33, 1813-1820 (1994).

20. M. S. Alam and M. A. Karim, "Multiple target detection using a modified fringe-adjusted joint transform correlator." Opt. Eng. 33, 1610-1617 (1994).

21. M. S. Alam, "Fractional power fringe-adjusted joint transform correlation," 34, 3208-3216 (1995).

22. T. Nomura, Y. Yoshimura, K. Itoh, and Y. Ichioka, "Incoherent-only joint-transform correlator," Appl. Opt. 34, 1420-1425 (1995).

23. T. Nomura, "Phase-encoded joint transform correlator to reduce the influence of extraneous signals," Appl. Opt. 37, 36513655 (1998).

24. I. Labastida, A. Carnicer, E. Martin-Badosa, I. Juvells, and S. Vallmitjana, "On-axis joint transform correlation based on a four-level power spectrum,” Appl. Opt. 38, 6111-6116 (1999).

25. H.-J. Su and M. A. Karim, "Phase-shifting joint transform correlation with phase-iterative algorithm: effect of the dynamic range limit," Appl. Opt. 39, 5556-5559 (1999).

26. A. Cherri and M. S. Alam. "Reference phase-encoded fringeadjusted joint transform correlation," Appl. Opt. 40, 12161225 (2001).

27. T.-C. Poon and A. Korpel, "Optical transfer function of an acousto-optic heterodyning image processor," Opt. Lett. 4, 317-319 (1979).

28. A. W. Lohmann and W. T. Rhodes, "Two-pupil synthesis of optical transfer functions," Appl. Opt. 17, 1145-1151 (1978).

29. T.-C. Poon. "Scanning holography and two-dimensional image processing by acousto-optic two-pupil synthesis," J. Opt. Soc. Am. A 2, 621-627 (1985).

30. J. Mait, "Pupil-function design for complex incoherent spatial filtering," J. Opt. Soc. Am. A 4, 1185-1193 (1987).

31. A. Korpel, "Acousto-Optics," in Applied Solid State Science, R. Wolfe, ed., Vol. 3 (Academic, New York, 1972).

32. T.-C. Poon and P. P. Banerjee, Contemporary Optical Image Processing With Matlab (Elsevier Science, Oxford, U.K., 2001).

33. B. Y. Soon, M. S. Alam, and M. A. Karim, "Improved feature extraction by use of a joint wavelet transform correlator," Appl. Opt. 37, 821-827 (1998). 